\section{Risk factors for physical inactivity across the adult life span: the impact of depression}

\author{
J Walker, H Christensen, T Windsor, A George \\ Centre for Mental Health Research, ANU, Canberra, Australia
}

\begin{abstract}
Aim: To determine the relative importance of sociodemographic characteristics, physical health and psychosocial functioning as correlates with physical inactivity across adulthood.

Methods: Data were collected from a population-based study of 7485 participants in three cohorts aged 20-24, $40-44$ and $60-64$ years. The prevalence of physical inactivity was determined for each age group. Data were gathered on potential risk factors for physical inactivity from sociodemographic, health and psychosocial functioning domains, with the aim of determining whether psychosocial functioning, especially depression, was an important correlate of physical inactivity after accounting for sociodemographic and health variables.
\end{abstract}

Results: The rates of physical inactivity increased with age, with $42.5 \%$ of younger and $53.8 \%$ of older adults classified as physically inactive. The importance of various correlates of physical inactivity differed across adulthood, with chronic physical conditions such as diabetes [odds ratio $(\mathrm{OR})=1.52, P<0.05$ ) and health behaviours such as current smoking $(\mathrm{OR}=1.75, P<0.001)$ being the strongest correlates for older adults. For younger and middle-aged adults, sociodemographic variables such as being unemployed $(P<0.05)$ and fewer years of education $(P<0.001)$ were correlates of physical inactivity. In terms of psychosocial functioning, depression $(P<0.01)$ remained a significant correlate of inactivity across all age groups, even after accounting for sociodemographic and health variables.

Conclusion: Depression is an important correlate of physical inactivity across the adult life span even after considering sociodemographic factors, health and lifestyle behaviours, and physical health.

\section{Factors influencing the decision to learn 5-HTT genotype results and subsequent impact on the individual}

\section{Kay Wilhelm, Bettina Meiser, Philip Mitchell, Jennifer Siegel, Tara Showyin, Gordon Parker, Peter R Schofield}

School of Psychiatry, University of NSW and Black Dog Institute, Sydney, Australia
Background: We reported an association between the 5-HTT gene and onset of MDD following exposure to adverse life events in a longitudinal cohort of postgraduate teacher trainees (Wilhelm et al. 2006). Many cohort members expressed interest in learning of their genotype results. With permission from the UNSW HREC, cohort members were given the opportunity to learn of their results and we investigated the reasons members did or did not want to know their results and how they received the knowledge.

Method: The 128 members who had genetic testing were sent measures prior to receiving their results covering: attitudes, perceived benefits and limitations to genetic testing; causal attributions to and perceived risk of depression onset; information needs and positive and negative affect. Follow up questionnaires were conducted at 2-week and 3-month follow up.

Results and Conclusions: Of the 116 responding members, $82(71 \%)$ indicated that they wanted their results, $22(19 \%)$ declined but agreed to complete the follow up questionnaires and 12 did not wish to participate. More members expected to have the $\mathrm{s} / \mathrm{s}$ genotype than was the case. There were no differences in rates of lifetime MDD diagnosis, but receivers had a later onset, fewer episodes, higher mean neuroticism scores and there was a trend towards more family MD history. All were glad to have received their genetic results and the perceived risk to depression fell across all genotype groups, with the greatest reduction for those with the $\mathrm{s} / \mathrm{s}$ genotype. Implications for research and the general community will be discussed.

\section{Understanding altered neural synchrony in first-episode schizophrenia}

\section{L (Lea) Williams' ${ }^{1}$, E Gordon ${ }^{1,2}$, A Harris ${ }^{1,3}$, P Das ${ }^{1,4}$,} W Wong ${ }^{1,5}$, G Flynn ${ }^{1,5}$, D Alexander ${ }^{2}$, T Whitford ${ }^{1}$

${ }^{1}$ Brain Dynamics Centre; ${ }^{2}$ Brain Resource International Database; ${ }^{3} T$ The University of Sydney; ${ }^{4}$ NISAD; and 5 Liverpool Hospital, Sydney, Australia

Background: First-episode schizophrenia (FES) is characterized by psychotic symptoms as well as profound difficulties in cognition and emotion. Our integrative neuroscience model of FES highlights the lack of coordinated neural synchrony underlying these features. The objective was to identify the cognitive, affective and neural synchrony markers that best differentiate FES from healthy controls, and the combination of markers that predict functional outcome.

Methods: We tested 56 FES (within 3 months of service contact) and 112 matched healthy controls as part of the Brain Resource International Database. Testing 